\title{
Fatores de risco e proteção para vínculos familiares no sul do Brasil
}

\author{
Vera Regina Rohnelt Ramires' \\ Universidade do Vale do Rio dos Sinos, RS, Brasil \\ Denise Falcke \\ Universidade do Vale do Rio dos Sinos, RS, Brasil
}

\begin{abstract}
Resumo: Este estudo buscou analisar fatores que protegem e que ameaçam os vínculos familiares em um município de uma região metropolitana no sul do Brasil. O delineamento foi transversal, descritivo e explicativo. Participaram da pesquisa 193 famílias em situação de vulnerabilidade social. Utilizou-se instrumentos que avaliaram recursos do ambiente familiar, rede de apoio, eventos adversos, estresse parental e problemas de saúde mental. Os resultados evidenciaram que em I2,6\% das famílias houve rompimento de vínculos. A maior parte da amostra, mesmo em condições de pobreza e de vulnerabilidade, mostrou-se capaz de preservar os vínculos familiares. Variáveis como escolaridade, condições de trabalho e renda, bem como condições de moradia, não foram associadas ao rompimento. As variáveis que se associaram, de modo significativo, foram estresse parental, problemas de ansiedade e depressão. Concluiu-se que as famílias apresentaram dinâmicas complexas, demandando a compreensão de múltiplos fatores envolvidos no rompimento de vínculos, especialmente relacionados à saúde mental.
\end{abstract}

Palavras-chave: família; vínculos familiares; saúde mental; risco; proteção.

\section{RISK AND PROTECTIVE FACTORS FOR FAMILY BONDS IN SOUTHERN BRAZIL}

\begin{abstract}
This study aimed at investigating factors that protect and risk family bonds in a metropolitan area in southern Brazil. We developed a cross-sectional, descriptive, and explanatory research, in which the sample was 193 socially vulnerable families. We used instruments to assess resources of the family environment, support network, adverse events, parental stress, and mental health problems. The results showed a bond break in $12.6 \%$ of the families. Most of the sample, even in conditions of poverty and vulnerability, was able to preserve family ties. There was not an association of variables such as education, working conditions, income, and living conditions with the break of bonds. The variables that associated, significantly, were parental stress and anxiety problems and depression. We concluded that the families had complex dynamics, requiring an understanding of multiple factors involved in breaking bonds, especially those related to mental health.
\end{abstract}

Keywords: family; family bonds; mental health; risk; protection.

FACTORES DE RIESGO Y PROTECCIÓN PARA VÍNCULOS FAMILIARES EN EL SUR DE BRASIL

Resumen: Este estudio ha investigado los factores de protección y riesgo para los lazos familiares en área metropolitana en el sur de Brasil. El diseño ha sido transversal, descriptivo y explicativo. Participaron 193 familias en situación de vulnerabilidad social.

${ }^{1}$ Endereço de correspondência: Vera Regina Rohnelt Ramires: Rua Luzitana, 1148, apt. 1002, Porto Alegre, Rio Grande do Sul, Brasil. CEP 90520-080.E-mail: verareginaramires@gmail.com 
Se evaluó los recursos del entorno familiar, red de apoyo, eventos adversos, estrés y problemas de salud mental. Los resultados han mostrado que en el I2,6\% de los hogares, hubo rompimiento de los lazos. La mayor parte de la muestra, incluso en condiciones de pobreza y vulnerabilidad, resultó capaz de preservar lazos familiares. La educación, condiciones de trabajo y de ingresos no se asociaron con la ruptura. Las variables asociadas fueran estrés de los padres y problemas de ansiedad y depresión. Se concluyó que las familias tenían dinámica compleja, que requiere una comprensión de los múltiples factores que intervienen en la ruptura de los lazos, especialmente relacionados con la salud mental.

Palabras clave: familia; lazos familiares; salud mental; riesgo; protección.

\section{Introdução}

Este estudo focalizou fatores de proteção e de risco para os vínculos familiares em um município de uma região metropolitana no sul do Brasil. O estudo buscou oferecer subsídios para a análise e o aprimoramento da implantação do Plano Municipal de Promoção, Proteção e Defesa do Direito de Crianças e Adolescentes à Convivência Familiar e Comunitária. Esse Plano, aprovado pelos Conselhos Municipais dos Direitos da Criança e do Adolescente (COMDEDICA) e de Assistência Social (CMAS), em 2009, constitui um desdobramento do Plano Nacional de Promoção, Proteção e Defesa do Direito de Crianças e Adolescentes à Convivência Familiar e Comunitária (PNDDCACF), política pública do governo federal aprovada em 2006, numa iniciativa do Conselho Nacional dos Direitos da Criança e do Adolescente (CONANDA) e do Conselho Nacional de Assistência Social (CNAS) (Brasil, 2006; São Leopoldo, 2009). O Plano Nacional e o Municipal constituem um marco nas políticas públicas do país, na medida em que buscam romper com a cultura da institucionalização e promover o paradigma da proteção integral e preservação dos vínculos familiares e comunitários.

Tais politicas preconizam que crianças e adolescentes sejam vistos de forma indissociável de seu contexto. Suas estratégias, objetivos e diretrizes orientam, prioritariamente, a prevenção do rompimento dos vínculos familiares, a qualificação dos serviços de acolhimento e o investimento no retorno ao convívio com a família de origem. Somente quando forem esgotadas essas possibilidades é que se deve recorrer ao recurso de encaminhamento para a família substituta, mediante procedimentos legais que garantam a defesa do melhor interesse da criança (Brasil, 2006; São Leopoldo, 2009).

$O$ desenvolvimento das crianças e dos adolescentes é continuamente afetado pelo contexto. Desde o entorno familiar, e a partir do ingresso na escola, da relação com colegas, professores, vizinhos, assim como a utilização das ruas, praças, igrejas, postos de saúde e outros espaços comunitários, formam-se grupos de relacionamento que constroem e ampliam seus vínculos afetivos (Cefai \& Camilleri, 2015; Savage, 2014). Na relação com a comunidade, instituições e demais espaços sociais, crianças e adolescentes se deparam com o coletivo, os papéis sociais, as regras, os valores, a cultura, as crenças e as tradições, transmitidos de geração para geração. Nesses espaços, constroem suas subjetividades e encontram recursos importantes para seu desenvolvimento.

A literatura já evidenciou a importância e a centralidade dos vínculos afetivos estabelecidos entre as crianças e os principais cuidadores e descreveu as necessidades 
essenciais das crianças: relacionamentos estáveis e contínuos; proteção física e segurança; regras e estabelecimento de limites; experiências que respeitem as diferenças individuais; experiências adequadas ao desenvolvimento; organização e expectativas; ambientes estáveis, amparadores e de continuidade cultural (Lima et al., 2014; Newland, 2015; Smokowski, Bacallao, Cotter, \& Evans, 2014). Quando a convivência é saudável, a família é o melhor lugar para o desenvolvimento da criança e do adolescente.

No entanto, é preciso avançar na compreensão das dificuldades que as famílias em situação de vulnerabilidade social enfrentam para oferecer esse ambiente às suas crianças e aos seus adolescentes (Coutinho, Matijasevich, Scazufca, \& Menezes, 2014). As necessidades de sobrevivência, as condições precárias de habitação, saúde e escolarização, além da exposição a ambientes de alta violência urbana são alguns dos fatores que explicam tais dificuldades (Coutinho et al., 2014; Gonçalves et al., 2014).

A saúde mental dos pais também tem sido considerada um importante fator de impacto para o desenvolvimento infantil (Guo et al., 2014; Lins \& Alvarenga, 2015; Van der Sluis, Van Steensel, \& Bögels, 2015; Teodoro, Hess, Saraiva, \& Cardoso, 2014). No estudo de corte realizado por Guo et al. (2014), com 577 mulheres de Ghana e Côte d'Ivoire, escores de estresse parental foram significativamente mais altos para mães deprimidas do que sem depressão. Na mesma direção, pesquisa realizada na Holanda por Van der Sluis, Van Steensel e Bögels (2015), com 68 pais de crianças, entre 4 e 12 anos, com sintomas de ansiedade, e 106 pais de crianças em grupo controle, evidenciou que os pais de crianças com sintomas de ansiedade reportaram parentalidade mais ansiosa (reforçando maior dependência e punição). Como consequência ao desenvolvimento infantil, o estresse parental e a baixa expectativa dos pais constituíram-se fatores de risco para problemas sociais, emocionais e comportamentais nos filhos em pesquisa realizada por Cefai e Camilleri (2015), com 486 crianças em Malta. O mesmo se observou em estudo com 2617 adolescentes estadunidenses em que as práticas parentais estiveram fortemente associadas à saúde mental dos adolescentes, repercutindo em maiores níveis de ansiedade, depressão e agressividade, assim como baixa autoestima e pior satisfação escolar (Smokowski et al., 2014). Também já foram identificadas repercussões em longo prazo do apego e das práticas parentais (Lima et al., 2014; Savage, 2014).

A literatura nacional corrobora os achados internacionais, evidenciando que a saúde mental dos cuidadores, especialmente da mãe, repercute nos sintomas psicológicos dos filhos. Estudo realizado com 197 adolescentes e seus pais (Teodoro, Hess, Saraiva, \& Cardoso, 2014) evidenciou que os problemas internalizantes e externalizantes maternos correlacionaram-se positivamente com sintomas internalizantes e externalizantes dos adolescentes, bem como com a percepção de um clima familiar negativo (mais conflito e menos afetividade). Na mesma direção, pesquisa realizada por Lins e Alvarenga (2015) com 103 mães evidenciou que o controle crítico materno, uma das dimensões do controle psicológico, repercutiu em retraimento e ansiedade/ depressão dos filhos. 
Ainda que a associação entre diferentes fatores pessoais, familiares e sociais já tenha sido comprovada pela literatura, são necessários estudos que favoreçam o mapeamento da interação entre os diferentes fatores de risco e proteção para a manutenção ou o rompimento dos vínculos familiares (Gonçalves et al., 2014). Sendo assim, os objetivos deste estudo foram: 1) identificar e correlacionar fatores que protegem e fatores que ameaçam os vínculos familiares em uma região metropolitana no sul do Brasil; e 2) identificar variáveis associadas à manutenção ou ao rompimento dos vínculos familiares na mesma região.

\section{Método}

O estudo teve delineamento quantitativo, transversal, descritivo e explicativo. Com base na literatura revisada, foram analisadas as seguintes variáveis relacionadas aos fatores de risco: eventos adversos, estresse parental e problemas de saúde mental. As seguintes variáveis foram analisadas como fatores de proteção dos vínculos familiares: recursos do ambiente familiar e percepção de rede e apoio social.

\section{Participantes}

Os participantes foram famílias de um município de região metropolitana do sul do Brasil em situação de vulnerabilidade social. Foram consideradas famílias em situação de vulnerabilidade social aquelas que enfrentam condições sociais, culturais ou relacionais adversas ao cumprimento de suas responsabilidades e/ou cujos direitos encontram-se ameaçados ou violados, conforme definição do PNDDCACF. Essas famílias constavam do Cadastro Único para Programas Sociais, instrumento que identifica e caracteriza famílias com renda mensal de até meio salário-mínimo por pessoa ou de três salários-mínimos no total, e eram beneficiárias do Programa Bolsa Família.

O município pesquisado possuía cerca de 8.800 famílias participantes do Programa Bolsa Família no período em que o Cadastro foi consultado. Nesse Cadastro, cada família tem um "Responsável pela Unidade Familiar", RF, que é um dos componentes da família, com idade mínima de 16 anos, preferencialmente mulher. Com base no número de famílias cadastradas, foi prevista a participação de 374 que tivessem pelo menos um filho em idade escolar, selecionadas por amostragem aleatória simples. 0 cálculo amostral teve como referência um nível de confiança de $95 \%$ e erro amostral de $5 \%$. As famílias eram pertencentes às regiões dos cinco Centros de Referência de Assistência Social (CRAS) da Secretaria Municipal de Assistência, Cidadania e Inclusão Social (SACIS).

\section{Instrumentos}

Questionário de Dados sobre a Família - Elaborado especificamente para este estudo, continha três blocos de questões: 1) dados gerais sobre a família, pessoas que residem na casa, escolaridade, ocupação, relacionamentos conjugais, condições da 
moradia, entre outros; 2 ) problemas de saúde física e mental de cada um dos moradores da residência; 3) situações que implicaram em rompimento de vínculos familiares, como separações, morte, mudanças, intervenções do Conselho Tutelar por violência doméstica, negligência e abandono, entre outros.

Inventário de Recursos do Ambiente Familiar (IRAF) (Maturano, 2006) - Baseado na concepção ecológica do desenvolvimento, o instrumento abrange três domínios: recursos que promovem processos proximais (oportunidades de interação com os pais, experiências estimuladoras do desenvolvimento, uso adequado do tempo livre, entre outros), atividades que sinalizam estabilidade na vida familiar (rotinas e reuniões regulares da família, cooperação da criança em tarefas domésticas) e práticas parentais que promovem a ligação família-escola. Maturano (2006) refere pesquisas que indicaram que o IRAF tem mostrado índices aceitáveis de fidedignidade (consistência interna medida pelo alfa de Cronbach variando de 0,70 a 0,84) e estabilidade da medida global com percentuais de acordo entre duas aplicações que variaram de $92 \%$ a $100 \%$ em três casos analisados.

Medida de Rede e Apoio Social (MRAS) - Divide-se em dois blocos; um deles avalia a rede social e o outro, o apoio social percebido, em quatro dimensões: apoio material (provisão de recursos práticos e ajuda material), apoio emocional (expressões de afeto positivo, compreensão e sentimentos de confiança), interação social positiva (disponibilidade de pessoas para se divertir e relaxar) e informação (disponibilidade de pessoas para obtenção de conselhos ou orientações). Traduzido e adaptado para o Brasil, obteve bons resultados de consistência interna, estabilidade e validade de constructo (Griep, Chor, Faerstein, \& Lopes, 2003). Conforme os autores, as dimensões de apoio social apresentaram consistência interna (coeficiente Alpha de Cronbach) variando entre 0,75 e 0,91 no teste e entre 0,86 e 0,93 no reteste. O coeficiente de correlação intraclasse variou entre 0,78 e 0,87 nas cinco dimensões da escala, sem diferenças importantes segundo o sexo, a idade ou o nível de escolaridade.

Escala de Eventos Adversos (EEA) - Formada por 36 itens descritivos de eventos adversos que podem ter ocorrido nos últimos 12 meses ou anteriormente na vida de uma criança, no contexto familiar. Divide-se em três subescalas: vida familiar, vida pessoal e vida escolar. A pontuação máxima é 72 , indicando grande quantidade de eventos adversos. Um estudo de fidedignidade através do procedimento teste-reteste forneceu índices de 100\%, 97\% e 94\% de estabilidade entre aplicações feitas com 20 dias de intervalo (Maturano, 2006).

Escala de Estresse Parental, EEP - Visa medir o nível de estresse dos pais como resultado de ter filhos. Consiste em 18 itens que descrevem a relação pai-filho e os sentimentos dos pais considerando essa relação. A pontuação máxima é 90 e, quanto mais próximo desse número for o resultado, mais elevado é o nível de estresse. A EEP é considerada apropriada para mães e pais de crianças com ou sem problemas clínicos (Berry \& Jones, 1995). Foi validada no Brasil por Brito e Faro (2017), que realizaram análise de confiabilidade (coeficientes variando de 0,90 a 0,97). Atestaram ainda 
evidências de validade concorrente da EEP com a Escala de Estresse Percebido (PSS-14) e o Inventário de Práticas Parentais (IPP).

Adult Self-Report for Ages 18-59 (ASR) - Composto por questões que avaliam dados pessoais, profissionais e de instrução, mais 126 itens sobre problemas de saúde mental (Achenbach, \& Rescorla, 2003). Esses itens identificam indicadores de problemas em várias áreas: ansiedade e depressão, isolamento, queixas somáticas (considerados problemas internalizantes), comportamento agressivo, quebra de regras e exibicionismo (considerados problemas externalizantes) e outros como problemas de pensamento e de atenção. Escores acima de 70 são indicadores de problemas clínicos. Esse instrumento, nas suas versões para as várias faixas etárias, é um dos mais utilizados mundialmente para rastrear problemas de saúde mental. Vem sendo extensivamente utilizado no Brasil e dados brasileiros foram relatados no estudo multicultural de validação do questionário, desenvolvido por Ivanova et al. (2015).

\section{Procedimentos de coleta de dados}

Mediante consulta ao Cadastro Único para Programas Sociais, foram identificadas famílias residentes na região vinculadas a cada um dos CRAS. Elas foram sorteadas e contatadas pela equipe de pesquisa por intermédio das escolas em que seus filhos estudavam (a frequência das crianças à escola é uma das condicionalidades do Programa Bolsa Família). Cada RF recebeu o convite para participar da pesquisa. Quando aceitaram e compareceram, foram esclarecidos sobre o estudo e assinaram o Termo de Consentimento Livre e Esclarecido (TCLE). Cada RF foi atendida(o) individualmente por entrevistadores treinados e responderam aos instrumentos na escola dos seus filhos ou na sede dos CRAS, em sala cedida para essa finalidade.

\section{Procedimentos de análise dos dados}

Foram realizadas as seguintes analises estatísticas: análises descritivas (médias, desvio-padrão, porcentagens) das variáveis em geral; análises comparativas, por meio do teste do qui-quadrado; análises de correlação de Pearson e análise de regressão logística. Para a seleção de variáveis da regressão logística foi utilizado o critério de $\mathrm{p}<0,20$ e utilizado o nível de significância de $5 \%$. Foi utilizado o pacote estatístico SPSS 20.0 .

\section{Procedimentos éticos}

O estudo foi aprovado pelo Comitê de Ética em Pesquisa da universidade à qual se vinculam as pesquisadoras e seguiu as normas das Resoluções 466/2012 do Conselho Nacional de Saúde. Todos os cuidados éticos foram respeitados. Situações de risco foram devidamente encaminhadas para o setor competente da rede de proteção, assegurando-se o bem-estar e a preservação dos participantes. 


\section{Resultados}

Participaram do estudo 193 famílias, por intermédio dos RFs. Após o sorteio das famílias, foram excluídas aquelas que não tinham filhos em idade escolar, as que não aceitaram participar do estudo ou as que, mesmo aceitando, não completaram os encontros necessários para responder aos instrumentos previstos. A Tabela 1 caracteriza os participantes quanto ao vínculo com a(s) criança(s), estado civil, escolaridade, profissão e renda.

Como se pode observar, em mais de $80 \%$ das famílias, as mães eram as Responsáveis pela Unidade Familiar. Mais de $60 \%$ não trabalhava fora, tinha o primeiro grau incompleto e uma condição socioeconômica precária, uma vez que mais de $80 \%$ desses participantes não tinham renda fixa ou tinham uma renda de até um salário-mínimo. Em torno de $40 \%$ não tinham companheiro(a).

A idade dos participantes variou de 19 a 74 (média $=39,85, D P=11,03$ ), o número de filhos variou de 1 a 12 filhos (média $=3,13, D P=1,80$ ). O número de cômodos na casa dos respondentes variou de 1 a 12 (média $=4,59, D P=1,49$ ). Cento e trinta e nove participantes $(73,2 \%)$ declararam ter enfrentado a separação de algum membro da família, sendo em 76 casos (40\%) de companheiro/marido, em 24 casos (12,6\%) de filho(a)s, em 21 casos $(11,1 \%)$ de mãe ou pai e em 18 casos $(9,5 \%)$ de outras pessoas.

Setenta e três participantes $(38,8 \%)$ declararam ter tido outro(s) companheiro(s) anteriormente. Em 120 casos (63,1\%), havia uma ou mais pessoas em casa com problemas de saúde. Dos 182 participantes que responderam à questão acerca de ser praticante de alguma religião, 170 responderam afirmativamente para regularmente ou às vezes ( $44 \%$ e 49,5\%, respectivamente), enquanto 12 respondentes declararam não ter prática religiosa $(6,6 \%)$. A Tabela 2 apresenta aspectos descritivos dos resultados obtidos com os instrumentos utilizados. 
Tabela I. Caracterização dos participantes quanto ao vínculo com a(s) criança(s), estado civil, escolaridade, profissão e renda

\begin{tabular}{|c|c|c|}
\hline Variável & $N$ & $\%$ \\
\hline \multicolumn{3}{|l|}{ Vínculo com a criança } \\
\hline Mãe & 159 & 82,8 \\
\hline Pai & 6 & 3,1 \\
\hline Avó & 18 & 9,4 \\
\hline Tia & 3 & 1,6 \\
\hline Outro & 6 & 1,6 \\
\hline \multicolumn{3}{|l|}{ Estado civil } \\
\hline Casada & 58 & 30,2 \\
\hline Com companheiro & 56 & 29,2 \\
\hline Solteira & 43 & 22,4 \\
\hline Separada & 26 & 13,5 \\
\hline Viúva & 9 & 4,7 \\
\hline \multicolumn{3}{|l|}{ Escolaridade } \\
\hline Sem instrução formal & 12 & 6,3 \\
\hline Ensino Fundamental incompleto & 118 & 61,8 \\
\hline Ensino Fundamental completo & 27 & 14,1 \\
\hline Ensino Médio incompleto & 19 & 9,9 \\
\hline Ensino Médio completo & 14 & 7,3 \\
\hline Ensino Superior incompleto & 1 & 0,5 \\
\hline \multicolumn{3}{|l|}{ Trabalha fora } \\
\hline Sim & 68 & 35,4 \\
\hline Não & 124 & 64,6 \\
\hline \multicolumn{3}{|l|}{ Profissão } \\
\hline Do lar & 110 & 57,6 \\
\hline Empregada doméstica & 26 & 13,6 \\
\hline Industriário & 8 & 4,2 \\
\hline Outras profissões (30 profissões) & 47 & 24,6 \\
\hline \multicolumn{3}{|l|}{ Salário } \\
\hline Sem renda fixa & 101 & 54,9 \\
\hline I salário mínimo & 59 & 32,1 \\
\hline 2-3 salários mínimos & 21 & $\mathrm{I} \mathrm{I}, 4$ \\
\hline 3-4 salários mínimos & 3 & 1,6 \\
\hline
\end{tabular}

Fonte: Elaborada pelas autoras. 
Tabela 2. Aspectos descritivos dos instrumentos utilizados com os responsáveis pelas unidades familiares

\begin{tabular}{|c|c|c|c|c|}
\hline Instrumento & Mínimo & Máximo & Média & $D P$ \\
\hline IRAF & 16 & 102 & 53,34 & 15,99 \\
\hline MRAS-afetivo-emocional & 7 & 75 & 28,95 & 9,56 \\
\hline MRAS-material & 4 & 20 & 13,36 & 4,75 \\
\hline MRAS-informação & 4 & 20 & 13,83 & 4,33 \\
\hline MRAS-interação social positiva & 4 & 32 & $|4,9|$ & 5,16 \\
\hline MRAS-total & 25 & |41 & 71,05 & 20,41 \\
\hline EEA-escolar & 0 & 8 & 3,01 & 1,82 \\
\hline EEA-familiar & 0 & 29 & 9,80 & 5,32 \\
\hline EEA-pessoal & 0 & 8 & 2,16 & 1,76 \\
\hline EEA-total & 0 & 35 & 14,96 & 6,81 \\
\hline EEP-total & 20 & 67 & 38,79 & 9,88 \\
\hline ASR-Isolamento & 0 & 15 & 6,71 & 2,97 \\
\hline ASR-Queixa somática & 0 & 21 & 6,93 & 4,78 \\
\hline ASR-Problemas de pensamento & 0 & 19 & 4,69 & 3,24 \\
\hline ASR-Problema de atenção & 0 & 26 & 9,05 & 4,22 \\
\hline ASR-Comportamento agressivo & 0 & 23 & 8,72 & 5,08 \\
\hline ASR-Quebra de regras & 0 & 13 & 3,48 & 2,74 \\
\hline ASR-Exibicionismo & 0 & 9 & 2,57 & 1,83 \\
\hline ASR-Ansiedade e depressão & 3 & 34 & 15,78 & 6,47 \\
\hline
\end{tabular}

Nota: IRAF = Inventário de Recursos do Ambiente Familiar, MRAS = Medida de Rede e Apoio Social, EEA = Escala de Eventos Adversos, EEP = Escala de Estresse Parental, ASR $=$ Adult Self-Report.

Fonte: Elaborada pelas autoras.

De acordo com o IRAF, os participantes relataram realizar atividades com as crianças, como acompanhar as atividades escolares, entre outras. Perceberam sua rede de apoio social, no geral, como sempre disponível. Os eventos adversos não se constituíram como uma ocorrência significativa para a maior parte da amostra, assim como o estresse parental, embora, nesse caso, um subgrupo apresentou índices mais elevados dessa variável. Finalmente, quanto aos indicadores de problemas de saúde mental, avaliados pelo ASR, o resultado mais elevado ocorreu na subescala de ansiedade e depressão, situado na faixa considerada clínica. 
Foram exploradas possíveis comparações entre a condição dos vínculos do participante, identificada com base no "Questionário de Dados sobre a Família" (variável dicotomizada em manutenção x rompimento de vínculos, considerando as famílias que vivenciaram ou não a separação dos filhos, por motivos de maus-tratos, negligencia ou abandono), e as variáveis sociodemográficas coletadas. Por meio do teste qui-quadrado, não foram encontradas diferenças entre as famílias com vínculos preservados ou rompidos com os filhos no que se refere às variáveis escolaridade $(\chi 2=1,38$, $g l=5, p=0,926)$, trabalho $(\chi 2=0,06, g l=1, p=0,795)$, estado civil $(\chi 2=1,10, g l=4$, $p=0,894)$, prática religiosa $(\chi 2=0,96, g l=2, p=0,618)$, moradia $(\chi 2=0,73, g l=3$, $p=0,866)$, ter tido outros companheiros $(\chi 2=1,40, g l=1, p=0,237)$ e ter pessoas em casa com problemas de saúde $(\chi 2=0,12, g l=1, p=0,837)$. Essas variáveis, portanto, foram excluídas de análises posteriores.

Buscando analisar associações entre as variáveis estudadas (recursos do ambiente familiar, rede e apoio social, eventos adversos, estresse parental e problemas de saúde mental - sintomas internalizantes e externalizantes), foram realizadas análises de correlação de Pearson. A Tabela 3 mostra as análises de correlação dos escores totais dos instrumentos respondidos pelos RFs.

\section{Tabela 3. Análises de correlação nos escores totais do IRAF, MRAS, EEA, EEP e ASR}

\begin{tabular}{lccccc} 
& 2 & 3 & 4 & 5 & 6 \\
\hline I. IRAF-total & $0,16^{*}$ & $-0,04$ & $-0,02$ & $-0,08$ & $-0,08$ \\
2. MRAS-total & - & $-0,02$ & $-0,16^{*}$ & $-0,21 *$ & $-0,11$ \\
3. EEA-total & & - & $0,22^{*}$ & $0,33 * *$ & $0,34^{* *}$ \\
4. EEP-total & & & - & $0,38^{* *}$ & $0,41^{* *}$ \\
5. ASR-Internalização & & & - & $0,65^{* *}$ \\
6. ASR-Externalização & & & & - \\
\hline
\end{tabular}

Nota: IRAF = Inventário de Recursos do Ambiente Familiar, MRAS = Medida de Rede e Apoio Social, EEA = Escala de Eventos Adversos, EEP $=$ Escala de Estresse Parental, ASR $=$ Adult Self-Report. ${ }^{*} p<0,05 * * p<0,0$ I.

Fonte: Elaborada pelas autoras.

De modo geral, os recursos do ambiente familiar apresentaram correlação positiva e de baixa magnitude com aspectos da rede de apoio social $(r=0,16)$, e relação negativa com a quantidade total de eventos adversos $(r=-0,16)$ e o estresse parental $(r=-0,21)$. Todas as correlações entre as variáveis de eventos adversos, estresse parental, problemas internalizantes e problemas externalizantes foram positivas e significativas, variando de $r=0,22$ a $r=0,65$.

Visando analisar a possibilidade de predição da manutenção ou rompimento dos vínculos com os filhos, realizou-se uma regressão logística utilizando a variável dependente vínculo (manutenção x rompimento) e os preditores idade, número de filhos, 
rede e apoio social (total de MRAS), recursos do ambiente familiar (total de IRAF), eventos adversos (total de EEA), estresse parental (total de EEP) e problemas de saúde mental (subescalas do instrumento ASR - Ansiedade/Depressão, Isolamento, Queixa Somática, Problemas de Pensamento, Problemas de Atenção, Comportamento Agressivo, Quebra de Regras, Exibicionismo). O modelo se mostrou significativo, com o teste omnibus $[\chi 2=37,89, g l=14, p=0,001]$, sendo corretamente classificados $87,5 \%$ dos casos.

Observou-se significância estatística para as variáveis Idade $[B=0,06, \operatorname{Exp}(B)=1,06$, $p=0,036]$, total de Estresse Parental $[B=0,08, \operatorname{Exp}(B)=1,08, p=0,010]$, e Ansiedade e Depressão $[B=0,14, \operatorname{Exp}(B)=1,15, p=0,050]$. Dessa forma, 0 aumento de um ponto no escore das variáveis Idade, Estresse Parental e Ansiedade e Depressão esteve relacionado a aumentos de $6 \%, 8 \%$ e $15 \%$ na probabilidade de ocorrer rompimento de vínculos, respectivamente.

\section{Discussão}

A análise dos resultados evidenciou que a maior parte das famílias estudadas, em que pese suas condições de pobreza e de vulnerabilidade, foi capaz de manter os vínculos afetivos com suas crianças. Das 193 famílias participantes, apenas 12,43\% vivenciaram a separação de seus filho(a)s, por razões relacionadas à negligência, maus-tratos ou abandono. Sem dúvida, trata-se de parcela importante que demanda atenção, tal como preconizam as políticas públicas (Brasil, 2006; São Leopoldo, 2009). No entanto, esses resultados atestam que a situação de pobreza e de vulnerabilidade não torna as famílias incompetentes para o cuidado e a criação dos filhos.

$\mathrm{Na}$ amostra estudada, a escolaridade, as condições de trabalho e de renda, o estado civil e o histórico conjugal, a religiosidade, as condições de moradia e ter pessoas em casa com problemas de saúde não foram associadas ao rompimento de vínculos. Situações de pobreza relacionadas à baixa escolaridade, ausência de trabalho, moradias precárias e famílias monoparentais já foram associadas na literatura ao rompimento de vínculos (Siqueira \& Dell'Aglio, 2011; D'Aroz, Stoltz, \& Martins, 2015). Todavia, assim como nos estudos encontrados, os fatores socioeconômicos não são suficientes para explicar tal fenômeno.

Na mesma direção, as análises de correlação mostraram que a presença de eventos adversos na vida das crianças e de estresse parental não se associou à existência de mais ou menos recursos no ambiente familiar. Entretanto, a ocorrência de eventos adversos foi associada de maneira significativa ao estresse parental e aos indicadores de problemas de saúde mental apresentados pelos cuidadores, confirmando dados da literatura nacional e internacional (Guo et al., 2014; Lins \& Alvarenga, 2015; Van der Sluis, Van Steensel, \& Bögels, 2015; Teodoro, Hess, Saraiva \& Cardoso, 2014). Por outro lado, diferentemente de outros estudos (Griep, Chor, Faerstein, \& Lopes, 2003; Pinto, Garcia, Bocchi, \& Carvalhães, 2006), nessa amostra, a percepção de rede de apoio não constituiu fator de proteção dos vínculos familiares. 
A análise de regressão logística evidenciou variáveis que se mostraram como preditoras do rompimento de vínculos. Esses resultados mostraram a idade do responsável, o estresse parental e as patologias relacionadas à ansiedade e à depressão como os fatores de risco mais significativos para o rompimento de vínculos, por abandono, negligência e abuso físico, entre os familiares e as crianças da amostra estudada.

É possível levantar a hipótese de que, diante de fatores como patologias relacionadas à ansiedade e depressão e estresse parental, principalmente, a presença de rede de apoio não seja suficiente para proteger a família e impedir a ruptura dos vínculos com os filhos. Tais rupturas, em geral, são resultantes de situações extremas e complexas, que demandariam mais do que uma rede de apoio para que fossem evitadas.

No entanto, é necessário ter cautela ao interpretar tais resultados e não incidir no equívoco de individualizar os problemas e responsabilizar as famílias, de forma descontextualizada, quando seus vínculos são rompidos. O artigo 23 do ECA (Brasil, 2009) preconiza que a falta ou a carência de recursos materiais não constitui motivo para perda ou suspensão do poder familiar. Indica, sim, a necessidade de inclusão dessas famílias em programas sociais. Por vezes, com excessiva rapidez, se decide retirar a criança da situação de pobreza, abandono ou violência para espaços que assegurem melhoras materiais de habitação e alimentação. Porém, é necessária uma mudança de paradigma (Ayres, Cardoso, \& Pereira, 2009), superando práticas de proteção da infância autoritárias, assistencialistas ou minimalistas.

Considera-se que as ações dirigidas às famílias para as quais o PNDDCACF se destina não podem ser reduzidas às suas condições socioeconômicas. Os resultados obtidos, tanto com as famílias que mantêm seus vínculos quanto com aquelas que enfrentaram rupturas, revelaram a presença de dinâmicas complexas, requerendo a análise e a compreensão dos múltiplos fatores envolvidos (Cefai \& Camilleri, 2015; Coutinho et al., 2014; Newland, 2015), bem como intervenções que focalizem diferentes dimensões.

A desigualdade social e a pobreza contribuem para que crianças e adolescentes sejam vítimas da fome, da ausência de abrigo ou de moradias precárias, falta de escolas e agressão física e sexual. Sua cronificação contribui para a deterioração das relações afetivas e parentais, gerando situações estressantes e danos ao desenvolvimento infantil. Entretanto, a carência de recursos materiais não é suficiente para explicar com profundidade o fenômeno do rompimento de vínculos e da violação dos direitos da criança e do adolescente.

Questões de saúde mental, como o estresse parental e os sintomas de ansiedade e de depressão, foram preponderantes na manutenção ou no rompimento de vínculos. Esse resultado corrobora estudos prévios (Guo et al., 2014; Lima et al., 2014; Teodoro, Hess, Saraiva, \& Cardoso, 2014; Van der Sluis, Van Steensel, \& Bögels, 2015) e evidencia a necessidade de atenção e acompanhamento da saúde mental dos responsáveis pelas unidades familiares.

Devem-se apontar alguns fatores limitadores deste estudo, como a perda amostral em relação ao número de famílias inicialmente previsto e as dificuldades para coletar os dados, em função das características dos participantes (percalços para comparecer, 
para responder/preencher instrumentos), além de serem residentes de um único município. Também foi utilizada uma variável de desfecho dicotômica, baseada em uma única questão. Contudo, os resultados deixaram claro que a garantia dos direitos de crianças e de adolescentes brasileiros à convivência familiar e comunitária não se esgota na esfera de uma única área: direitos humanos, assistência social, saúde, educação. A complexidade dessa política transcende os limites de disciplinas específicas, bem como de estruturas político-administrativas particulares, como ministérios e secretarias estaduais ou municipais. É imperativo que gestores dos diferentes setores superem suas diferenças, para que os programas e as ações possam ser pensados e planejados em conjunto, sendo colocados em prática de forma articulada e cooperativa.

A literatura já destacou que o trabalho para a superação das vulnerabilidades das famílias deve abarcar suas condições de miserabilidade (incluindo condições de habitação, segurança alimentar, geração de trabalho e renda), suas formas de relacionar-se e vivenciar diferentes papéis e responsabilidades (rompendo ciclos de violência, muitas vezes transgeracionais) e sua integração sociocomunitária, garantindo acesso a serviços públicos e a rede de apoio (Siqueira \& Dell'Aglio, 2011; Nery, 2010). Este estudo evidenciou que, além de tais dimensões, atenção especial deve ser dedicada às condições de saúde dessas famílias, especialmente de saúde mental. A exposição aos eventos adversos, o estresse ligado aos papéis parentais e os sintomas indicativos de sofrimento psíquico se constituíram, nesta amostra, como uma ameaça à preservação dos vínculos familiares. Na implementação do PNDDCACF, essas variáveis poderiam ser tomadas como indicadores de risco e orientar o trabalho da rede de proteção e de saúde, no sentido da prevenção e da garantia dos direitos das crianças e adolescentes à convivência familiar e comunitária.

\section{Referências}

Achenbach, T. M., \& Rescorla, L. A. (2003). Manual for the ASEBA adult forms \& profiles. Burlington, VT: University of Vermont, Research Center for Children, Youth, and Families.

Ayres, L. S. M, Cardoso, A. P., \& Pereira, L. C. (2009). O abrigamento e as redes de proteção para a infância e a juventude. Fractal: Revista de Psicologia, 21(1), 125-136. doi:10.1590/S1984-02922009000100010

Berry, J. O., \& Jones, W. H. (1995). The Parental Stress Scale: Initial Psychometric Evidence. Journal of Social and Personal Relationships, 12, 463-472. doi:10.1177/0265407595123009

Brasil (2006). Plano Nacional de Promoção, Proteção e Defesa do Direito de Crianças e Adolescentes à Convivência Familiar e Comunitária. Brasília: Ministério do Desenvolvimento Social e Combate à Fome, Secretaria Especial de Direitos Humanos. 
Brasil (2009). Estatuto da Criança e do Adolescente - ECA, Lei 12.010, sancionada em 03 de agosto de 2009 altera a Lei 8.086, de 13 de julho de 1990. Brasília, DF: Diário Oficial da União (DOU). Recuperado de http://www.planalto.gov.br/ ccivil_03/_Ato2007-2010/2009/Lei/L12010.htm

Brito, A., \& Faro, A. (2017). Diferenças por sexo, adaptação e validação da Escala de Estresse Parental. Avaliação Psicológica, 16(1), 38-47.

Cefai, C., \& Camilleri, L. (2015). A healthy start: promoting mental health and well-being in the early primary school years. Emotional and Behavioural Difficulties, 20(2), 133-152. doi:10.1080/13632752.2014.915493

Coutinho, L. M. S., Matijasevich, A., Scazufca, M., \& Menezes, P. R. (2014). Prevalência de transtornos mentais comuns e contexto social: análise multinível do São Paulo Ageing \& Health Study (SPAH). Cadernos de Saúde Pública, 30(9), 1875-1883. doi:10.1590/0102-311X00175313

D'Aroz, M. S., Stoltz, T., \& Martins, P. C. (2015). A vez e a voz de mulheres-mães com filhos institucionalizados: Narrativas de histórias de vida sobre a vida em família Niemcy: Novas Edições Académicas.

Gonçalves, D. A., Mari, J. D. J., Bower, P., Gask, L., Dowrick, C., Tófoli, L. F., Campos, M., ... Fortes, S. (2014). Brazilian multicentre study of common mental disorders in primary care: rates and related social and demographic factors. Cadernos de Saúde Pública, 30(3), 623-632. doi:10.1590/0102-311X00158412

Griep, R., Chor, D., Faerstein, E., \& Lopes, C. (2003). Apoio social: confiabilidade teste-reteste de escala no Estudo Pro-Saúde. Cadernos de Saúde Pública, 19(2), 625-634. doi:10.1590/S0102-311X2003000200029

Guo, N., Bindt, C., Te Bonle, M., Appiah-Poku, J., Tomori, C., Hinz, R., Barthel, D., ... Ehrhardt, S. (2014). Mental health related determinants of parenting stress among urban mothers of young children-results from a birth-cohort study in Ghana and Côte d'Ivoire. BMC psychiatry, 14(1), 156. doi:10.1186/1471-244X$14-156$

Ivanova, M. Y., Achenbach, T. M., Rescorla, L. A., Turner, L. V., Ahmeti-Pronaj, A., Au, A., Avila Maese, C., ... Zasepa, E. (2015). Syndromes of self-reported psychopathology for ages 18-59 in 29 societies. Psychopathology and Behavioral Assessment, 37(2), 171-183. doi:10.1007/s10862-014-9448-8

Lima, A. R., Mello, M. F., Andreoli, S. B., Fossaluza, V., de Araújo, C. M., Jackowski, A. P., Bressan, R. A., \& Mari, J. J. (2014). The impact of healthy parenting as a protective factor for posttraumatic stress disorder in adulthood: a case-control study. PLOS ONE, 9(1), e87117. doi:10.1371/journal.pone.0087117

Lins, T., \& Alvarenga, P. (2015). Controle psicológico materno e problemas internalizantes em pré-escolares. Psicologia: Teoria e Pesquisa, 31(3), 311-319. 
Marturano, M. (2006). O Inventário de Recursos do Ambiente Familiar. Psicologia: Reflexão e Crítica, 19(3), 498-506. doi:10.1590/S0102-79722006000300019

Nery, M. A. (2010). A convivência familiar e comunitária e direito da criança e do adolescente e uma realidade a ser repensada pela escola. Cad. Cedes, Campinas, 30(81), 189-207.

Newland, L. A. (2015). Family well-being, parenting, and child well-being: Pathways to healthy adjustment. Clinical Psychologist, 19(1), 3-14. doi:10.1111/cp.12059

Pinto, J. L. G, Garcia, A. C. O, Bocchi, S. C. M, \& Carvalhães, M. A. (2006). Características do apoio social oferecido a idosos de área rural assistida pelo PSF. Ciência \& Saúde Coletiva, 11(3), 753-764. doi:10.1590/S1413-81232006000300023

São Leopoldo (2009). Plano Municipal de Promoção, Proteção e Defesa do Direito de Crianças e Adolescentes à Convivência Familiar e Comunitária. São Leopoldo: Secretaria Municipal de Assistência, Cidadania e Inclusão Social.

Savage, J. (2014). The association between attachment, parental bonds and physically aggressive and violent behavior: A comprehensive review. Aggression and violent behavior, 19(2), 164-178. doi:10.1016/j.avb.2014.02.004

Siqueira, A. C., \& Dell'Aglio, D. D. (2011). Políticas públicas de garantia do direito à convivência familiar e comunitária. Psicologia \& Sociedade, 23(2), 262-271. doi:10.1590/S0102-71822011000200007

Smokowski, P. R., Bacallao, M. L., Cotter, K. L., \& Evans, C. B. (2014). The Effects of Positive and Negative Parenting Practices on Adolescent Mental Health Outcomes in a Multicultural Sample of Rural Youth. Child Psychiatry \& Human Development, 46(3), 333-345. doi:10.1007/s10578-014-0474-2

Teodoro, M. L. M., Hess, A. R. B., Saraiva, L. A., \& Cardoso, B. M. (2014). Problemas emocionais e de comportamento e clima familiar em adolescentes e seus pais. Psico (Porto Alegre), 45(2), 168-175.

Van der Sluis, C. M., Van Steensel, F. J. A., \& Bögels, S. M. (2015). Parenting clinically anxious versus healthy control children aged 4-12 years. Journal of anxiety disorders, 32, 1-7. doi:10.1016/j.janxdis.2015.03.002 